\title{
MathBlackBoard as Effective Tool in Classroom
}

\author{
Deguchi Hiroaki ${ }^{1}$ and Hashiba Hirokazu ${ }^{2}$ \\ ${ }^{1}$ Kobe University, 2-1 Rokkodai-cho, Nada-ku, Kobe 657-8501, Japan \\ 2 Akashi Junior High School attached to Kobe University, \\ 3-4 Yamashita-cho, Akashi 673-0878, Japan
}

\begin{abstract}
MathBlackBoard is a Java program that has unique GUI (Graphical User Interface). By using GUI, MathBlackBoard users can input, edit and manipulate mathematical expressions easily. And MathBlackBoard has its own kernel. However, it has functions to use other computer algebra system as computational engine.

In this paper, we describe an example of using MathBlackBoard. We have added a new function which is designed to be used in junior high school classroom. And we have used it in classrooms.
\end{abstract}

\section{Introduction}

MathBlackBoard is based on a Java applet named "blackboard applet" which was written in 1997 by Matsushima J. ([1]). The blackboard applet has a GUI where users can input, edit and manipulate mathematical expressions easily. And GUI of the blackboard applet is inherited to MathBlackBoard. Moreover, a kernel of MathBlackBoard is developed from some objects of the blackboard applet.

The purpose of the blackboard applet was to create an "ease to use" computer algebra system. Matsushima argued that difficult matters about the educational use of computer algebra systems are "difficulties of input by using CUI (Character-based User Interface or Command-line User Interface)" and "dependence to OS." The blackboard applet was produced to try to resolve these issues. "GUI buttons for mathematical operation" and "drag \& drop with the meaning of mathematical operation" were mentioned as resolutions to the first issue. And second issue has been resolved by "use of Java applet on web browser."

\section{GUI of MathBlackBoard}

We have improved the blackboard applet after the master thesis([1]), and decided to rename it MathBlackBoard ([2] [3]). Now MathBlackBoard is able to run not only as Java applet, but also as Java application. And it is reconstructed as a set of objects. Then, MathBlackBoard has been enriched with new functions which can connect to some computer algebra systems and use them as computational engine (4]). In addition, its GUI operations have been improved. 


\subsection{GUI Windows}

MathBlackBoard has three GUI windows: Blackboard window (Fig. 11), Keyboard window and GraphScreen window. Blackboard window is main window that has editing space. Mathematical expressions are shown in this window. And users can input, edit and manipulate expressions on Blackboard window.

Keyboard window is used to input and edit expressions. And MathBlackBoard provides GraphScreen window where graphs are drawn.

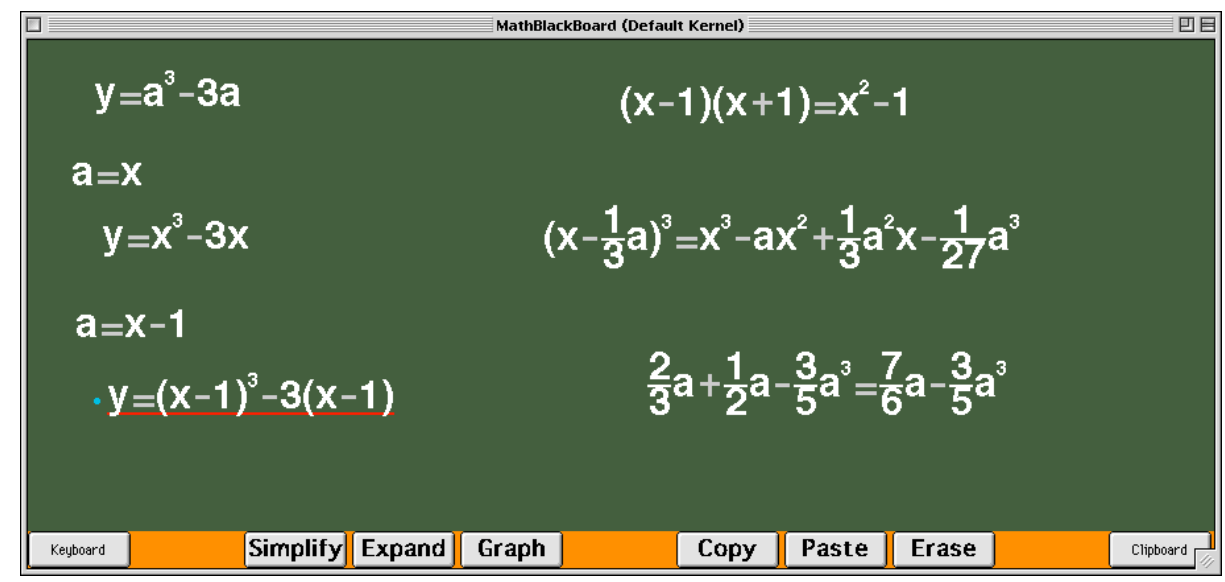

Fig. 1. Blackboard Window

The function buttons are placed at the bottom of Blackboard window (Fig. 11). These GUI buttons are provided to execute functions instead of typing commands.

\subsection{Drag \& Drop}

One of characteristics of MathBlackBoard is flexibility of position of mathematical expressions displayed. Expressions can be moved to anywhere on the editing area of Blackboard window. And MathBlackBoard provides "drag \& drop" to manipulate mathematical expressions. Editorial operations and mathematical operations are assigned to "drag \& drop."

Editorial operations are as follows: Move expression to another point, Connect grabbed expression to the other expression, Copy selected expression to clipboard, and Paste an expression from clipboard.

Mathematical operations are as follows: Substitute, Draw graph, and Perform functions assigned to GUI buttons.

\section{Using MathBlackBoard in Classroom}

We have had an opportunity of testing MathBlackBoard in classrooms, at Akashi Junior High School attached to Kobe University. The classes were in the unit 


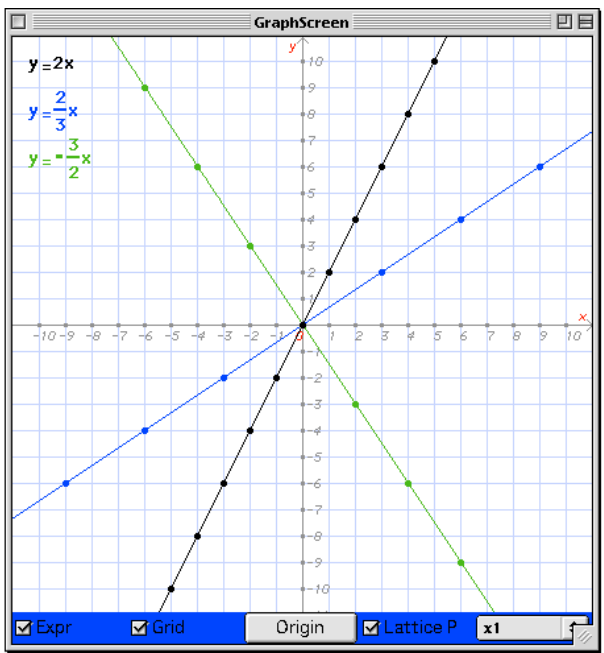

Fig. 2. New GraphScreen Window for the Class

"direct \& inverse proportion." MathBlackBoard has been used in the lessons to make graphs of direct proportion. The purposes of the lesson were as follows:

1. To understand the difference of graphs between plus-constant and minusconstant of direct proportion

2. To make graphs of direct proportion of fraction-constant, by using pen and paper

\subsection{Preparations for the Class}

The teacher of the classes requested to improve MathBlackBoard as follows:

1. The range of GraphScreen window must be "-10 to 10 " which is about the same of student's graph paper

2. Mathematical expressions of graphs are shown by default

3. Grid lines are drawn by default

4. Lattice points of graphs are described, if it could be

It is the reason for the request " 4 " that students of the classes had learned to make graphs by connecting dots. The teacher expected that students read out graphs on MathBlackBoard strictly, and compare them with graphs on students' paper.

\subsection{Scenes on the Class}

MathBlackBoard was used as follows:

1. The teacher present how to make a graph of direct proportion by using MathBlackBoard

2. By using pen and paper, students make graphs of some expressions presented 
3. Students use MathBlackBoard, to make graphs of presented expressions

4. Students compare graphs of their paper with graphs of MathBlackBoard

\section{Conclusion}

With those implementation above, MathBlackBoard has been students' easily accessible tool. Students were able to acquire how to use MathBlackBoard after teacher's presentation. The teacher presented how to use MathBlackBoard just once per a class. It was enough time for students to acquire how to use MathBlackBoard. They were able to grade their graphs on paper easily.

After these classes, we discussed about improvement of MathBlackBoard. The teacher remarked that he required new functions. And he showed a plan to use these new functions in other scene.

We consider "substitution by using drag \& drop" can help student's understanding of concept of substitution. But it is necessary to bring MathBlackBoard close to the "pen and paper" style. We have to implement new style similar to student's work in their notebook, instead of mechanical replacement.

The goal of MathBlackBoard development is to provide new environment. It is not an environment that solve problems, but an environment where users can solve problems.

\section{References}

1. Matsushima J.: Useful Computer Algebra System by Using Java. Master Thesis, Kobe University. (1998) [in Japanese]

2. Deguchi H.: Blackboard Applet, Journal of Japan Society for Symbolic and Algebraic Computation 9(1) (2002) 32-37 [in Japanese]

3. Deguchi H.: MathBlackBoard, Journal of Japan Society for Symbolic and Algebraic Computation 11(3,4) (2005) 77-88 [in Japanese]

4. Deguchi H.: MathBlackBoard as User Interface of Computer Algebra Systems, Proceedings of the 10th Asian Technology Conference in Mathematics (2005) 246-252 